\title{
La eVolución Patrimonial del Monasterio de Santa María de la Concepción de la orden de San Jerónimo de Granada (Ss.XV-XVII)
}

\author{
JuAn José SÁnchez CARRasco ${ }^{1}$ \\ Universidad de Granada
}

Recibido: 14 de febrero de 2018

Aceptado: 12 de marzo de 2018

\begin{abstract}
Resumen
El objetivo del presente trabajo es estudiar el desarrollo patrimonial del Monasterio de Santa María de la Concepción de la orden de San Jerónimo de Granada. Para la elaboración del ensayo se ha realizado una exhaustiva búsqueda documental en archivos tanto granadinos como nacionales y se han empleado los datos de las publicaciones existentes sobre el monasterio que datan de los años 90 del pasado siglo. Se ha conseguido realizar un estudio completo sobre todas las transacciones que realizó la orden durante los siglos XV-XVI y ubicar sus propiedades mediante Sistemas de Información Geográfica (SIG).
\end{abstract}

\section{Palabras clave}

Granada; Reyes Católicos; Orden de San Jerónimo; economía; siglos XV-XVI.

\begin{abstract}
The aim of this work is to study the heritage development of the Monastery of Santa Maria de la Concepción of the Order of Saint Jerome in Granada. For this purpose, an exhaustive data search has been undertaken in both local and national archives, in addition to the use of information contained in the literature published on the topic in the 90s of the twentieth century. It has been possible to achieve a comprehensive study of all the transactions carried out by the Order during the $15^{\text {th }}$ and $16^{\text {th }}$ centuries and locate their properties using geographic information systems (GIS).
\end{abstract}

\section{Keywords}

Granada; the Catholic Monarchs; Order of Saint Jerome; Economy; Fifteenth and Sixteenth Centuries.

\footnotetext{
1 Correo electrónico: j.j.sanchezc@hotmail.com. Orcid: https://orcid.org/0000-0002-3213-0112. Contratado predoctoral del Departamento de Historia Medieval y Ciencias y Técnicas Historiográficas de la Universidad de Granada. Financiado por el Ministerio de Educación, Cultura y Deporte. El presente trabajo forma parte del proyecto I+D: «La propiedad aristocrática en la Granada nazarí y su traspaso a la sociedad castellana después de la conquista (siglos XIII-XVI)» (HAR2015-64605-C2-2-P), que a su vez está integrado en un proyecto I+D coordinado titulado «Almunias del Occidente islámico». La finalidad es el estudio de almunias y otras fincas aristocráticas en al-Ándalus desde las perspectivas arqueológica, arquitectónica y documental.
} 


\section{Riassunto}

Lo scopo di questo lavoro è quello di studiare lo sviluppo del patrimonio del Monastero di Santa Maria de la Concepción dell'Ordine di San Girolamo di Granada (Spagna). Per l'elaborazione di questo articolo, è stata necessaria un grande lavoro di ricerca documentaria negli archivi locali e nazionali, e sono stati utilizzati anche pubblicazioni esistenti sul monastero, fatti dagli anni ' 90 del secolo scorso. È stato possibile ricostruire in toto le transazione effettuate dall'ordine fra i secoli XV-XVI e la distribuzione geo-spaziali delle proprietà mediante l'utilizzo di Geographic Information System (GIS).

\section{Parole chiavi}

Granada, Re Cattolici, Ordine di San Girolamo, economia, secoli XV-XVI.

\section{Introducción}

Los estudios económicos sobre las órdenes regulares asentadas a lo largo de la geografía peninsular siempre han estado muy presentes en la historiografía, no podemos obviar la importancia que los monasterios y conventos tuvieron en la sociedad medieval y moderna. En los últimos años y gracias a la incorporación a PARES de gran cantidad de documentación inédita proveniente de la sección Clero del Archivo Histórico Nacional, se han multiplicado los estudios sobre las órdenes monásticas y el tema vuelve a estar de moda, han apareciendo numerosos trabajos desde los enfoques más clásicos cómo los estudios económicos y patrimoniales a otros con nuevas perspectivas cómo la de género $^{2}$. Desde una perspectiva económica y enlazando con la temática del presente ensayo, las distintas ordenes (franciscanos, dominicos, jerónimos, cartujos, jesuitas, etc.) utilizaron diversas estrategias para atesorar una gran cantidad de bienes urbanos y rústicos, participaron de la Hacienda regia comprando juros y llegaron a configurar auténticos latifundios que se mantienen hasta la desamortización de Mendizábal. La única excepción la tenemos con la Compañía de Jesús que entre su fundación en 1534 y su expulsión de España en 1767, y a diferencia de otras órdenes, compró una gran cantidad de superficie agrícola de primera calidad en la que introdujeron novedades técnicas para fomentar la producción destinada al comercio 3 . No obstante, no todas las fundaciones monásticas tuvieron el mismo éxito, algunas casas no llegaron a prosperar y otras tuvieron una vida efímera ${ }^{4}$.

\footnotetext{
2 Sobre conventos femeninos y el estado de la cuestión actual podemos citar un excelente trabajo de Atienza LóPez, "El mundo de las monjas y de los claustros femeninos en la edad moderna", vol. 1, pp. 89108. Sobre conventos en Granada, marco geográfico donde se centra el presente estudio, podemos citar a García VALVERde, "El archivo del convento de agustinas recoletas del santísimo Corpus Christi de Granada" y García Valverde, "El monasterio de Santa Isabel la Real de Granada".

3 Sobre la Compañía de Jesús debemos mencionar los trabajos de LóPEZ MARTíneZ, "El patrimonio rústico de los jesuitas en España" y "El patrimonio económico de los jesuitas en el reino de Sevilla". Un estudio similar al aquí presentado, pero centrado en los cartujos fue realizado también por LóPEZ MARTínez "La empresa agraria monástica andaluza".

4 Sin ánimo de resultar exhaustivo podemos citar para la mencionada temática los trabajos de ATIENZA LÓPEZ, "Fundaciones frustradas y efímeras en la España moderna" y "Los que fracasaron: fundaciones y fundadores frustrados".
} 
Centrándonos ya en la orden de San Jerónimo, debemos puntualizar que tenía una relación muy cercana a la Corona de Castilla desde su fundación por bula papal el 18 de octubre de 1373. A finales del siglo XV ya existían 25 monasterio de la orden en la Península Ibérica, los distintos monarcas dotaron muchas fundaciones como la del Real Monasterio de Santa María de Guadalupe o el Real Monasterio de Nuestra Señora de Fresdelval. Los jerónimos se caracterizaron por no traspasar las fronteras de la península ibérica, siempre estuvieron muy vinculados a la monarquía castellana tal como hemos mencionado pero también a la Corona portuguesa ${ }^{5}$. Los monjes seguían la regla de San Agustín y su vida cotidiana se regía en un equilibrio entre la oración y el trabajo. Muchos de los monasterios donde habitaron, han superado los avatares de la desamortización y llegado hasta nuestros días, constituyendo un magnífico ejemplo del poder económico y político que tuvieron las órdenes monásticas a finales del medievo y durante la Edad Moderna ${ }^{6}$.

La orden de San Jerónimo se asentó en Granada en 1492 tras la finalización de la contienda. En los siglos posteriores se convirtió en el monasterio más rico e influyente de la antigua capital del emirato nazarí. No obstante, el estudio del monasterio de Santa María de la Concepción adolece de importantes lagunas. El motivo de ello es la destrucción de parte de los archivos de la orden durante la ocupación francesa y su posterior dispersión tras la desamortización de Mendizábal. En la actualidad existen fondos documentales pertenecientes al monasterio granadino bastante diseminados y depositados en diversos archivos ${ }^{7}$. A nivel historiográfico son pocas las publicaciones que versan sobre el monasterio, a excepción de un trabajo del arriba firmante y otro de José Rodríguez Molina más recientes, el resto de ensayos sobre el tema datan de la década de los 90 del pasado siglo. Sobre la cuestión han escrito Rafael Marín López, Manuel Espinar Moreno, Adelina Romero Martínez y Carmen Trillo San José. Las perspectivas de estudio empleadas por los mencionados autores han sido diversas y parciales, abordándose por ejemplo cuestiones meramente económicas como en los casos de Rafael Marín López y Manuel Espinar Moreno, artísticas en el trabajo de

\footnotetext{
Miembros de los jerónimos siempre han sido personajes relevantes en la Corona de Castilla, siendo parte de los distintos consejos e incluso ocupando cargos muy importantes, durante los siglos XV y XVI tenemos varios ejemplos de ello, en el año 1492 fray Nuño de Arévalo prior del Real Monasterio de Guadalupe de la Orden de San Jerónimo era miembro del Consejo Real. En 1516 Cisneros, encargó el gobierno de Indias a fray Luis de Figueroa prior del monasterio de Mejorada del Campo. Como último ejemplo podemos mencionar a fray Hernando de Talavera, confesor de la reina Isabel y primer arzobispo de Granada.

6 Para conocer el progreso general de la orden jerónima es de obligatoria consulta la obra de José SÁNCHEZ Herrero, "Fundación y Desarrollo de la Orden de los Jerónimos, 1360-1561".

7 Los fondos documentales del monasterio de Santa María de la Concepción están repartidos en varios archivos de la geografía española. En los archivos granadinos encontramos documentación en el Archivo de la Real Chancillería de Granada (ARCHG), en el Archivo histórico provincial de Granada (AHPG). En este último, el lib. 1252 correspondiente al Catastro de Ensenada, incluye numerosa información, no obstante, no ha sido incluido en su totalidad porque excede los límites cronológicos del presente ensayo. En archivos de ámbito estatal existen fondos depositados en el Archivo Histórico Nacional (AHN) y en el Archivo General de Simancas (AGS). Se tiene constancia de la existencia de documentación en el propio monasterio granadino. No obstante, su consulta es prácticamente imposible debido a que en la actualidad está habitado por religiosas de la orden jerónima que practican voto de clausura.
} 
Adelina Romero Martínez o sobre regiones concretas de la geografía granadina en la que la orden recibió bienes como recogen los ensayos de Carmen Trillo San José. Concretando los objetivos del presente estudio, se tratará de analizar las transacciones realizadas por el monasterio granadino durante los Ss. XV-XVII y que supusieron la formación de un inmenso patrimonio. Para ello se ha contado con las fuentes editadas y material inédito encontrado tras una revisión de los documentos editados así como un nuevo vaciado en diversos archivos ${ }^{8}$.

\section{Adquisiciones de tierra en la Vega de Granada y en Santa Fe: consolidación de un modelo latifundista (Ss. XV-XVII)}

Los inicios del monasterio de San Jerónimo fueron duros, si bien su presencia está documentada en el Reino de Granada desde 1492, los monjes vivieron brevemente en Santa Fe, en la ermita de Santa Catalina durante menos de un año. Posteriormente se trasladaron a la capital, a una huerta llamada la Almoraba, donde permanecieron hasta 1521 cuando se trasladaron definitivamente a la huerta de Dar Abenmordí. Fue en dicho lugar donde se construyó finalmente el monasterio y la iglesia. La Corona, para ayudar a la instalación y edificación, había realizado dos generosas donaciones, la primera en 1495 y la segunda en $1504^{10}$. El pago de algunas rentas no fue recibido por la orden o se hizo con problemas tras los primeros años de la conquista castellana. Los motivos de los impagos, pagos fraccionarios y demoras se debieron a varias circunstancias. No podemos obviar que la ciudad de Granada vivió una rebelión pocos años después de su capitulación, o que los efectos de la Guerra de Granada se dejaron notar en la economía no sólo del antiguo Reino nazarí, sino de toda la Corona de Castilla ${ }^{11}$. En ocasiones la propia hacienda regia, necesitada de fondos, embargó directamente el pago de los arrendatarios de la orden ${ }^{12}$. A todo ello debemos sumar una serie de malas cosechas acaecidas durante la primera década del siglo XVI, que conllevaron al incremento del precio del pan y a la escasez de cereal ${ }^{13}$. No obstante, desde la primera dotación real el monasterio contó con importantes bienes rústicos y urbanos ${ }^{14}$. El cobro de las rentas, que sí fueron percibidas, produjo una importantísima fuente de ingresos para las arcas

\footnotetext{
8 A los fondos documentales recogidos en los archivos citados en la anterior nota, debemos sumar una obra inédita a la que se ha tenido acceso gracias a la doctora María Luisa García Valverde. Dicho trabajo es un manuscrito escrito en 1975 por el desaparecido Manuel Garzón Pareja. La mencionada obra tiene un gran valor, ya que contiene transcripciones de documentación depositada actualmente en el Monasterio de San Jerónimo y a la que nadie más ha tenido acceso.

9 La voz "Dar" que en árabe significa casa, aparece en la documentación castellana sin traducción, en alguna ocasión se sustituye por "Dan".

10 Sánchez Carrasco, "Las dos dotaciones fundacionales del Monasterio de Santa María de la Concepción de Granada".

11 Vera Delgado, "La Revuelta Mudéjar de 1500-1501: El destino de los vencidos".

12 Marín López, "Origen y evolución del patrimonio del Monasterio de San Jerónimo de Granada", p. 224

13 Luna Díaz, "Apuntes para el estudio de la agricultura de Granada y su tierra en los primeros años de la conquista”, p. 243

14 Sánchez CARrasco, "Las dos dotaciones fundacionales...", pp. 512-520.
} 
del monasterio desde el mismo momento de su fundación, lo que provocó que inmediatamente comenzase a adquirir propiedades, tal como aparece recogido en las actas capitulares de la orden. Esto provocó la articulación del inmenso patrimonio del que hará gala la congregación a finales del siglo XVI.

Debemos puntualizar que el monasterio raramente vendió una propiedad, aunque en algún caso sí realizó alguna permuta para completar las que habían sido donadas por la Corona. La estrategia empleada por el monasterio granadino consistió en adquirir propiedades rentables, que pudiesen ser adquiridas a buen precio y arrendarlas en las distintas modalidades de censo comunes en la época. Las transacciones normalmente se pagaban al contado, también fue común que se incluyese como forma de pago animales de su cabaña ganadera, ya que los jerónimos granadinos fueron uno de los mayores poseedores de cabezas de ganado de la capital del antiguo emirato nazarí. Estas operaciones fueron de especial relevancia en los años inmediatamente posteriores a la conquista castellana, el motivo de la concentración de compras en este periodo es debido al bajo precio que tenía la tierra en estos momentos. Podemos añadir incluso que en algunos casos la orden llegó a pedir dinero prestado y a endeudarse para poder llevar a cabo algunas transacciones ${ }^{15}$.

Como ya hemos mencionado, la estrategia principal de la orden fue consolidar sus posesiones para posteriormente ir ampliándolas adquiriendo las huertas y hazas adyacentes, llegando de esta manera a configurar verdaderos latifundios. Un ejemplo clarísimo de ello lo tenemos en la propia Santa Fe. La ermita de Santa Catalina, primer lugar donde se asentó la orden, había quedado a su cuidado y para el mantenimiento de la misma se habían destinado 150 marjales que contaba con casa y huerto ${ }^{16}$.

En la primera mitad del siglo XVI, la orden realizó en Santa Fe quince operaciones que supusieron la adquisición de nuevas propiedades, entre las que encontramos compra, permuta, redención de censo y venta de censo. Las primeras compras se producen en 1501, nueve años después del traslado de la orden a la ciudad de Granada. Cabe mencionar que el monasterio en este momento aún no se había consolidado y pugnaba porque le entregasen la huerta de Dar Abenmordí, donde finalmente se ubicó ${ }^{17}$. No tener una sede fija, ni dinero para erigir la iglesia y el monasterio, tal como alegaron los monjes a la Corona, no supuso un contratiempo para que los hermanos ampliasen el patrimonio de la orden. En las dos primeras compras realizadas el citado año, la orden adquiere 105 marjales por un precio de 14.655 maravedíes, y posteriormente 42 marjales más realizando un desembolso de 4.284 maravedíes $^{18}$.

La compra de tierras se sigue sucediendo a lo largo de la primera mitad del siglo. En 1507, con el monasterio ya asentado definitivamente, adquieren 15 marjales por un im-

\footnotetext{
15 Rodríguez Molina, “Los jerónimos de Granada. Procedencia de sus riquezas”, p. 278.

16 Peinado Santaella, La fundación de Santa Fe, pp. 352-355.

17 SÁnchez CARrasco, "Las dos dotaciones fundacionales...", pp. 523-525.

18 Las operaciones realizadas por la orden en Santa Fe están recogidas en el Libro Becerro del convento de San Jerónimo de Granada (AHN), sección clero, lib. 3693.
} 
porte de 4.500 maravedíes. Podemos apreciar que el precio de la tierra había aumentado progresivamente a lo largo de los primeros años del siglo XVI. Diez años después en 1517, vuelven a realizar una adquisición, en esta ocasión adquieren 5 marjales por 2.000 maravedíes. En la década de los 30 del siglo XVI, la orden se mostró especialmente activa adquiriendo nuevas posesiones en Santa Fe. Encontramos entre estos años las primeras permutas entre propiedades. El año 1530, la orden cambia 10 marjales por 16, con una compensación de 4.875 maravedíes. En 1533 permuta 81 marjales por 51, en esta ocasión sin compensación económica, no conocemos si la otra parte de la transacción abonó algo al monasterio por los 30 marjales de diferencia, otra hipótesis que podemos arrojar es que los marjales recibidos por la orden fueran de mejor calidad que los entregados. Ya que en la documentación referida a Santa Fe, encontramos alusiones a la excelente calidad de la tierra en aquella zona ${ }^{19}$. En 1536 se produce otra compra, 10 marjales por 11.250 maravedíes. El mismo año se produce una redención de censo sobre tierras regidas por el monasterio con una compensación de 750 maravedíes. En 1537 y 1538 se producen dos nuevas permutas, ambas sin compensación económica. Se cambian 12 marjales por $10 \mathrm{y}$ posteriormente 10 por 13. En la década de los 40 se realizan menos transacciones, pero de mayor envergadura que en años anteriores. En 1542 la orden vende un censo sobre 11 marjales por 25 maravedíes. El año siguiente realizaron una nueva transacción, 8 marjales por 15.000 maravedíes. La adquisición que supuso un desembolso de mayor cuantía para las arcas de la orden se produjo el año 1546. Compran 91 marjales por la nada desdeñable cantidad de 170.625 maravedíes. Dos años después vuelven a comprar 10 marjales por 3.750 maravedíes y finalmente en 1551 adquieren 10,5 marjales por 27.368 maravedíes $^{20}$. Como hemos observado, en un periodo de cincuenta años, el monasterio de Santa María de la Concepción, tan sólo en el entorno de Santa Fe, realiza un desembolso de 254.575 maravedíes para adquirir 274,5 marjales a los que debemos sumar los 150 que ya poseía en la localidad. La superficie resultante equivale a algo más de 22 hectáreas, este es un claro ejemplo de la política de ampliación de propiedades realizada por la orden a la que hacíamos alusión anteriormente ${ }^{21}$.

En referencia a la gran diferencia que podemos observar en el precio que se pagó por marjal en cada una de las transacciones, puede deberse a varios factores. En primer lugar, las compras realizadas en los años inmediatamente posteriores a la conquista de la ciudad fueron realizadas a moriscos que vendían sus posesiones a precios bajos con el objetivo de pasar a allende, cerca de Santa Fe, en las alquerías de Chauchina y el Jau, Gómez de Santillán, alcaide de Santa Fe, adquirió a finales del siglo XV la totalidad de las tierras de labor a precio de saldo ${ }^{22}$. En los primeros años del siglo XVI

\footnotetext{
19 Entre los documentos incluidos en los apéndices del trabajo de Peinado Santaella, La fundación de Santa Fe. Encontramos numerosas alusiones a la excelente calidad de la tierra de en el entorno de la villa. 20 Marín López, “Origen y evolución...”, p. 229.

21 La equivalencia se ha realizado usando la superficie del marjal granadino establecido en $528.42 \mathrm{~m}^{2}$. Según Morell y Terry, Equivalencias métricas de la provincia de Granada, p. 65.

22 Peinado Santaella, "Una aportación documental sobre el poblamiento, el paisaje agrario y la propiedad de la tierra de dos alquerías de la vega de Granada".
} 
el precio del marjal seguía bajo y equivalía a unos 200 maravedíes $^{23}$. Aunque el precio aumentaba según la calidad de la tierra, si era de secano o de regadío y si había alguna infraestructura allí construida. Si atendemos a lo desembolsado por la orden en Santa $\mathrm{Fe}$, observamos como el precio medio del marjal es casi 5 veces superior a la media indicada, ya que la cantidad resultante es de 944.44 maravedíes por marjal. Por lo tanto, debemos suponer que las tierras adquiridas por la orden eran de buena calidad y regadío, además que posiblemente muchas de ellas tendrían casas, acequias u otras instalaciones, aunque desgraciadamente no tenemos suficiente documentación para confirmarlo.

\begin{tabular}{|c|c|c|c|}
\hline Año & No de Marjales & $\begin{array}{c}\text { Tipo de } \\
\text { transacción }\end{array}$ & Importe \\
\hline 1501 & 105 & Compra & 14.655 \\
\hline 1501 & 42 & Compra & 4.284 \\
\hline 1507 & 15 & Compra & 4.500 \\
\hline 1517 & 5 & Compra & 2.000 \\
\hline 1530 & 10 por 16 & Permuta & 4.875 \\
\hline 1533 & 81 por 51 & Permuta & - \\
\hline 1536 & 10 & Compra & 11.250 \\
\hline 1537 & 12 por 10 & Permuta & - \\
\hline 1538 & 10 por 13 & Permuta & - \\
\hline 1542 & 11 & Venta (censo) & +25 \\
\hline 1543 & 8 & Compra & 15.000 \\
\hline 1546 & 91 & Compra & 170.625 \\
\hline 1551 & 10,5 & Compra & 27.386 \\
\hline \multicolumn{4}{|r|}{} \\
\hline Total & 274,5 Marjales & Total & 254.575 Maravedís \\
\hline
\end{tabular}

Tabla de las transacciones realizadas en Santa Fe en la primera mitad del S. XVI ${ }^{24}$.

Una gran propiedad situada en la Vega que acabó en posesión del monasterio fue el denominado Cortijo de Ansola, ubicado cerca de Pinos Puente. El 15 de abril del año 1525 la duquesa de Sessa, Doña María Manrique, viuda de Gonzalo Fernández de Córdoba el Gran Capitán, fundó una capellanía en el monasterio ${ }^{25}$. Dicha fundación incluía 200 fanegas de trigo y 200 de cebada situadas en el mencionado cortijo ${ }^{26}$. En 1568 Gonzalo Fernández de Córdoba, nieto del Gran capitán vendió el cortijo a la orden por 29.000 maravedíes a cambio de que este sufrague la ornamentación de la

\footnotetext{
23 Rodríguez Molina, "Los jerónimos de Granada...", p. 270.

24 La tabla ha sido elaborada según los datos aportados por el manuscrito de GARZÓN PAREJA, La orden de San Jerónimo en Granada, el trabajo de MARín LóPEz, "Origen y evolución..." y el libro 3693 depositado en la Sección Clero del (AHN).

25 Gracias a las transcripciones incluidas en Garzón Pareja, La orden de San Jerónimo en Granada. Podemos conocer todas las capellanías que se fundaron en el monasterio desde su construcción hasta el siglo XVIII. Toda la información sobre el patronato del Gran Capitán fue publicada en la primera década del siglo pasado por Valladar y Serrano, "El Patronato del Gran Capitán en la Iglesia de San Jerónimo", y "La Iglesia de San Jerónimo".

26 Garzón Pareja, La orden de San Jerónimo en Granada, p. 92.
} 
capilla del monasterio de Nuestra Señora de la Concepción en Granada y condone las mandas testamentarias legadas por la ya difunta duquesa de Sessa ${ }^{27}$. En garantía de que el monasterio cumpliría con lo acordado entregaría el duque la posesión de las tiendas del Zacatín que rentaban 1.000 ducados anuales ${ }^{28}$. Las mencionadas tiendas, habían sido donadas al monasterio en la primera dotación real en $1495^{29}$. Sobre las tiendas de las cuales era propietario el monasterio, sabemos que ya en el siglo XVII, en el año 1636 para ser exactos, aún tenía 20 distribuidas por toda la ciudad que rentaban 135.156 maravedíes al año ${ }^{30}$. Lo cual nos permite recoger la trayectoria de algunos bienes donados a la orden casi 150 años después de que fuesen entregados al monasterio por los Reyes Católicos.

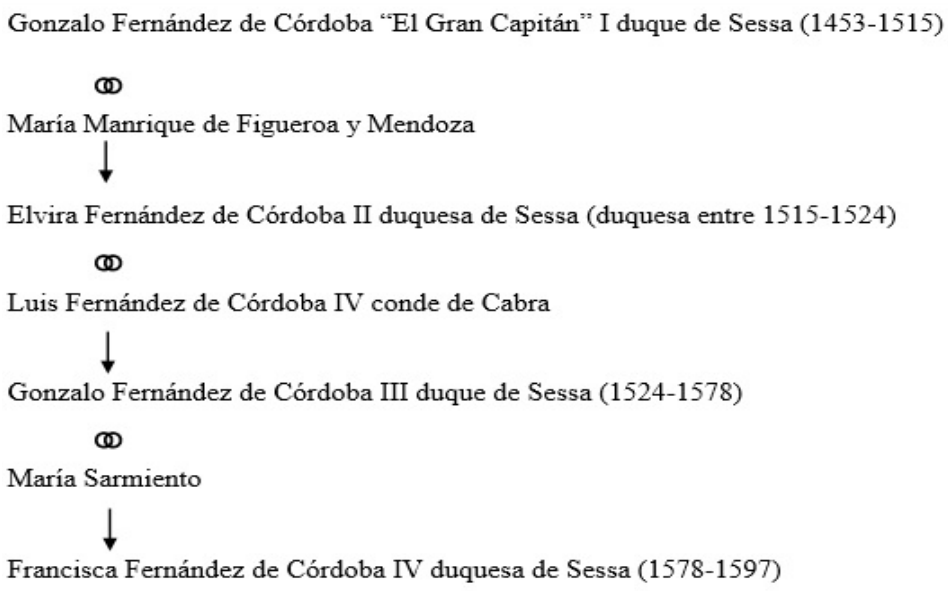

Relación de titulares del ducado de Sessa en los siglos XV y XVI ${ }^{31}$.

El Cortijo de Ansola, que actualmente tiene la misma denominación, está situado en la localidad de Pinos Puente en la zona noroccidental de la Vega de Granada y lindaba con el Cortijo de Çohaira. Fue entregado por la Corona al Gran Capitán por sus méritos durante la guerra una vez finalizó la contienda. Se trataba de una gran explotación agrícola con una superficie de unos 3.000 marjales. El cortijo rentaba unas 1000 fanegas de cereal al año. En la mayoría de las grandes propiedades del monasterio, los monjes construyeron

27 Marín López, “Origen y evolución...”, p. 233. La venta del Cortijo de Ansola supuso el despido de Diego de Siloé que en esos momentos trabajaba en el monasterio, Romero MarTínez, "Documentos para la historia del arte granadino. El Monasterio de San Jerónimo".

28 Rodríguez Molina, "Los jerónimos de Granada...”, p. 270. El valor del ducado castellano estaba fijado en 375 maravedíes desde el 13 de junio del año 1497 por la Real Pragmática de Medina del Campo. Por ende, la renta de las tiendas del Zacatín en la segunda mitad del S.XVI era de 375.000 maravedíes.

29 SÁnchez Carrasco, "Las dos dotaciones fundacionales...", pp. 512-513.

30 (AHN), Sección Clero, lib. 3693, folS. 125v-139r.

31 Fuente: Elaboración propia. 
infraestructuras para mejorar su rendimiento. El Cortijo de Ansola no fue una excepción, rápidamente construyeron presas para el riego en los ríos Velillos y Cubillas. Casas y cabañas fueron edificadas para los labradores y el ganado, lo cual nos indica que esta propiedad fue usada para el pastoreo de los rebaños del monasterio. También se construyó un horno para cocer ladrillos y tejas ${ }^{32}$. Podemos apreciar cómo los jerónimos tendían a diversificar las actividades económicas dentro de una misma propiedad.

Nuevamente apreciamos como los jerónimos trataron de concentrar propiedades en una misma zona. En el año 1568, a la par que adquirieron el Cortijo de Ansola, intentaron comprar también al duque de Sessa, nieto de Gonzalo Fernández de Córdoba, el denominado Cortijo del Coscojar, cerca del primero. Los monjes siempre estuvieron atentos al mercado para comprar propiedades a buen precio. En esta ocasión entraron en la puja por la mencionada propiedad ya que el cortijo se sacó en almoneda por una cantidad inferior a su valor real. La calidad de la tierra era "de lo mejor que ay en la Vega", aunque finalmente no se llevó a cabo la transacción. Parece ser que lo que subyacía bajo la venta del Cortijo de Ansola y de El Coscojar era la necesidad de dinero que en ese momento tenía el noble.

\section{Terratenientes al norte de Granada: el Cortijo de los Árboles, el Pago de Cújar y el Cortijo Navarrete (Ss. XV-XVII)}

La otra gran hacienda que conformó el estudiado monasterio fue la que tuvieron en la zona norte de Granada, una gran propiedad distribuida en los términos de Cújar, Víznar, Pulianas, Alfacar y Jun. Su formación comienza desde los primeros años del siglo XVI, en estas fechas se detecta la compra de pequeñas fincas o hazas a campesinos moriscos ${ }^{33}$. La formación del inmenso latifundio con tierras en los pagos de Víznar, Jun y Pulianas tuvo su epicentro el cortijo de Enciso-Navarrete. La propiedad se fraguó desde el inicio del siglo XVI, aunque desgraciadamente para este caso tenemos menos información que para las transacciones realizadas durante el mismo periodo de tiempo en Santa Fe. Entre la documentación manejada es común que no aparezcan el número de marjales que eran comprados por la orden, sólo aparece indicada la cantidad desembolsada por ella, por lo que lo único que podemos realizar es una estimación aproximada de la superficie total que el monasterio poseyó en esta ubicación.

La primera compra que se produce en la zona fue realizada en el año 1505, la propiedad comprada fue el Pago de Cújar. Tal cómo se indicó anteriormente no conocemos la cantidad de marjales que tenía esta propiedad. El vendedor fue el morisco Juan Abegladon y el precio de la compra fue de 54.375 maravedíes $^{34}$. La fecha es importante ya que durante ese mismo año la orden pleiteaba con Isabel Rebollo, viuda del licenciado Andrés Calderón, primer corregidor de Granada, por la posesión de la huerta de Dar

\footnotetext{
Rodríguez Molina, "Los jerónimos de Granada...", p. 270.

3 MARÍN LóPEz, “Origen y evolución...”, p. 229.

34 (AHN), sección Clero, lib. 3693, fol. 123r.
} 
Abenmordí, lugar donde se iniciaría la obra del monasterio ${ }^{35}$. Una de las alegaciones que había realizado la orden para justificar la demora en la construcción de la iglesia y monasterio era que andaba escasos de fondos ${ }^{36}$. Pues bien, en 1505, a los 54.375 maravedíes que pagaron por el Pago de Cújar debemos sumar los 94.000 que desembolsaron para hacer acopio de los materiales necesarios para los comienzos de la obra, lo que nos hace suponer que las arcas del monasterio no estaban tan vacías como decían los monjes $^{37}$. Poco a poco fueron ampliando esta propiedad comprando tierras anexas con dinero procedente de capellanías, limosnas o ventas de otras propiedades. Podemos citar como ejemplo la venta del horno de Guimien, entregado a la orden en la primera dotación de los Reyes Católicos de 1495, y que fue vendido en 1608 al Hospital de San Juan de Dios por 45.000 maravedíes. Este dinero fue invertido en la compra de una finca de olivo y vid que había pertenecido a Diego de Alcaraz ${ }^{38}$. Si atendemos a todas las operaciones realizadas por el monasterio en la zona de Cújar, observamos que entre el año 1505 y el año 1625 sólo en esta zona, se efectuaron 33 compras con un desembolso aproximado de 450.000 maravedíes. Desgraciadamente, y tal como se indicó con anterioridad, no conocemos la superficie que abarcaban las propiedades adquiridas por el monasterio ya que no aparece indicado en la documentación analizada ${ }^{39}$.

La primera gran compra que realizó el monasterio en la zona se produjo en 1525, fecha en la que los monjes están ya plenamente instalados y con abundantes ingresos. Por ello pudo adquirir el Cortijo de Árboles (La Granja) que contaba con una superficie de 12.247 marjales, por la cantidad de 375.000 maravedíes. En la transacción se incluyeron también 14 vacas, gallinas, patos, colmenas y palomas ${ }^{40}$. Aquí vemos tal y como se mencionó con anterioridad la utilización de animales de la cabaña ganadera del monasterio como medio de pago. El origen de parte de los fondos empleados para la adquisición del cortijo era procedente de la capellanía que Domingo Velastegui había fundado el 6 de febrero de 1521 dejando en herencia numerosos bienes y más de 230.000 maravedíes $^{41}$. La capacidad adquisitiva que demuestra el monasterio durante el primer cuarto del siglo XVI nos hace suponer que los jerónimos granadinos disponían ya de amplia solvencia económica. Si observamos la cantidad desembolsada, vemos que es prácticamente la misma o incluso un poco más de la totalidad de las rentas que percibían a principio de siglo tras las dos donaciones regias ${ }^{42}$.

El cortijo fue comprado al jurado de Granada Francisco Ortiz que lo había adquirido cuatro años antes, en 1521, y cuyos antiguos propietarios habían sido los moriscos Bernardino Minxiamixi, Alonso el Zarí y el mercader del Albaycín Juan Alahacén. Los tres lo habían

\footnotetext{
5 SÁnchez Carrasco, "Las dos dotaciones fundacionales...", pp. 529-530

36 SÁnchez CARrasco, "Las dos dotaciones fundacionales...”, pp. 529-530.

7 SÁnchez CARrasco, "Las dos dotaciones fundacionales...", p. 531.

8 MARÍn LóPez, “Origen y evolución...”, p. 232.

39 Las transacciones realizadas por la orden en la zona de Cújar, Árboles, Pulianas y Víznar están recogidas en el Libro Becerro del convento de San Jerónimo de Granada (AHN), sección clero, lib. 3693.

40 (AHN), Sección Clero, lib. 3693, fol. 208v.

41 Garzón Pareja, La orden de San Jerónimo en Granada, p. 98.

42 SÁnchez Carrasco, "Las dos dotaciones fundacionales...,", pp. 513-514 y pp. 526-527.
} 
comprado en 1517 al escribano de la Audiencia Juan Pérez de Barahona ${ }^{43}$. Estaba situado en el término de Colomera junto a la carretera de Jaén. La mayoría de la superficie era de secano, aunque contaba con algunas hazas de regadío cercanas al Rio Cubillas. Llegados a este punto, habremos de destacar que la compra-venta de propiedades no fue una actividad exclusiva de las élites castellanas. Este hecho podemos evidenciarlo con el caso del Cortijo de Árboles, la propiedad fue pasando por diferentes manos tanto castellanas como moriscas hasta su adquisición final por parte del monasterio.

La compra fue una buena operación ya que se realizó hizo por un valor inferior al que realmente tenía. La razón de ello es que estaba poblado de álamos y encinas, de manera que, aunque no hicieron grandes cambios en la propiedad, aprovecharon su riqueza forestal. En los 25 años posteriores a su adquisición, el cortijo fue roturado y se plantaron olivos, viñas y cereal, tónica común entre las tierras de secano adquiridas por los castellanos tras la conquista del Reino Nazarín ${ }^{44}$. La madera de los árboles fue utilizada para reformar el Cortijo de Ficullar, posesión de la orden ubicada en la región del Quempe ${ }^{45}$. Se edificaron diversos edificios para almacenar y procesar los distintos productos que allí se producían. Así, se construyó una bodega para el vino, una almazara de viga para el aceite junto con un almacén para su almacenaje, un granero y varias cabañas para el ganado. Esto nos indica que tanto las tierras del cortijo como las anexas, que también habían sido compradas por la orden conformando un extenso latifundio, eran empleadas para el pastoreo de su extensa cabaña ganadera. La presencia de ganado en el cortijo es lógica si atendemos la extensa zona de secano con la que contaba la mencionada propiedad y la cercanía de algunas dehesas que la orden poseía en la Sierra de Alfaguara ${ }^{46}$.

Aunque la compra del Cortijo de Árboles también supuso un pleito para el monasterio, en esta ocasión contra una institución tan influyente y poderosa como el Tribunal del Santo Oficio de la Inquisición. Poco tiempo después de su adquisición la Inquisición demandó la posesión de la finca debido a que el anterior propietario, el jurado de la ciudad de Granada Francisco de Ortiz había sido procesado y sentenciado por judaizante. Los jerónimos alegaron que no iban a entregar la propiedad ya que ellos habían comprado el cortijo antes que su antiguo dueño judaizase. Esto provocó un largo pleito de casi 25 años que al final ganaron los monjes.

\footnotetext{
43 Garzón Pareja, La orden de San Jerónimo en Granada, p. 130.

44 Trillo SAn José, "El paisaje vegetal en la Granada Islámica y sus transformaciones tras la conquista castellana".

45 El Quempe es una región situada al suroeste de Granada, zonas de esta región eran propiedad de los Infantes de Granada, Trillo SAN José, "Fiscalidad mudéjar en el reino de Granada: las rentas del Quempe", pp. 855-857. El cortijo de Ficullar fue donado a la orden por los Reyes Católicos en la primera dotación que le hicieron al monasterio, Juan José SÁNCHEZ CARRASCO, "Las dos dotaciones fundacionales...", p. 516. El mencionado cortijo también fue propiedad de los Infantes de Granada, (AHN), sección Clero, lib. 3693, fol. 209v. 46 Rodríguez Molina, "Los jerónimos de Granada...”, p. 270.
} 
Así: "el monasterio salió con el porque la venta se hizo mucho antes que judaizase y assi se dio sentencia en favor del monasterio y se trajo executoria de la inquisición de Valladolid donde se siguió el pleito, su fecha en Valladolid en diez de febrero de 1551 "'47.

Finalmente, el monasterio pidió una real provisión tras conocer la sentencia para enviársela al licenciado Lagunilla, fiscal real, que había puesto la demanda por el tema de la herejía del jurado Ortiz ${ }^{48}$. Lo que subyace bajo el pleito entre jerónimos y la Inquisición es meramente un tema económico. Debemos recordar que los bienes de todos los procesados por el Tribunal del Santo Oficio eran incautados y revertían en la Corona, única institución de la que la Inquisición era dependiente. Otra cuestión que debemos abordar, viendo los precedentes, es que el jurado Francisco de Ortiz hubiera vendido a tan buen precio el cortijo debido a que era consciente de que iba a ser procesado y así evitar la pérdida de su patrimonio, siendo el dinero mucho más fácil de ocultar que las propiedades. Sobre el Cortijo de Árboles podemos añadir que el alcalde mayor de Granada el doctor Sirviente a petición de los jerónimos amojonó los lindes de la finca el 1 de febrero de 1547 y que la propiedad pasó a ser conocida como La Granja ${ }^{49}$. Pese a que el monasterio había ganado el pleito contra la Inquisición, el cambio del nombre quizás sea debido al recelo que sentían por la lacra que suponía que el nombre del cortijo fuese vinculado a una persona procesada por la Inquisición y sentenciado por hereje judaizante.

Para realizar el análisis de cómo se fue ampliando esta propiedad en Árboles, tenemos más información que para las tierras de Cújar ${ }^{50}$. El monasterio llevó a cabo un total de siete operaciones entre el año 1527 y 1545 . La primera adquisición la hizo al morisco Alonso Çauça por 11.250 maravedíes dos años después de la adquisición del cortijo. En esta compra no aparece indicada la superficie de la finca, pero trataremos de hacer una aproximación a tenor del importe desembolsado por la orden en otras operaciones. En el año 1533 efectuó dos compras, ambas de 3 marjales, abonando 476 maravedíes a cada uno de los vendedores, Diego Gaçen y Juan Elgarire. En 1542 se realiza una permuta con el oidor de Granada Diego Peña Aranda, éste entrega al monasterio 50 fanegas de la medida de Córdoba (609 marjales) a cambio de "ciertos pedazos que aquel tenía en Sierra Elvira, dos potros y otras cosas" 51 . Conocemos el origen de las tierras que poseía el oidor granadino, las había recibido en 1513 por una real cédula del rey Fernando. En 1543 nuevamente se realiza una doble compra, Lorenzo Aliçaraque y Juan Albenarí venden cada uno 723 marjales por 9.375 maravedíes. Finalmente dos años después los jerónimos hacen la última transacción con propietarios de la zona, de nuevo es Lorenzo Aliçaraque el vendedor, que recibe 750 maravedíes por solo un marjal ${ }^{52}$.

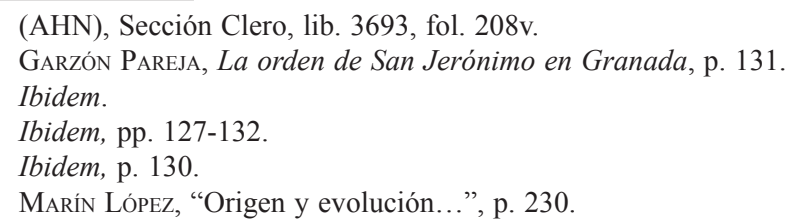


Como podemos apreciar por los nombres de los antiguos propietarios, la orden compra en esta región tierras principalmente a moriscos. Si sumamos las compras realizadas en Víznar, Jun y Alfacar a las que hemos visto en Cújar, vemos como en esta zona el monasterio invierte en algo más de un siglo 2.706.000 maravedíes en 58 transacciones $^{53}$. Aunque realizar un cálculo del total de la superficie adquirida es bastante complejo ya que debemos tener en cuenta que más de la mitad de los marjales de la zona eran de secano, lo cual implicaba que tuvieran menor valor que los de regadío y que el precio del marjal se vio incrementado a lo largo del siglo $\mathrm{XVI}^{54}$.

\begin{tabular}{|c|c|c|c|}
\hline Año & № de Marjales & Tipo de transacción & Importe \\
\hline 1505 & (Pago de Cújar) & Compra & 54.375 \\
\hline 1525 & 12.247 (Cortijo de Árboles) & Compra & 375.000 \\
\hline 1527 & - & Compra & 11.250 \\
\hline 1533 & 3 & Compra & 476 \\
\hline 1533 & 3 & Compra & 476 \\
\hline 1542 & 609 & Permuta & - \\
\hline 1543 & 723 & Compra & 9.375 \\
\hline 1543 & 723 & Compra & 9.375 \\
\hline 1545 & 1 & Compra & 750 \\
\hline \multicolumn{4}{|l|}{} \\
\hline Total (Conocido) & 14.309 & Total & 461.077 Maravedis \\
\hline
\end{tabular}

Tabla de transacciones conocidas al norte de Granada ${ }^{55}$.

La enorme propiedad fue ampliada en 1589 con una extensa superficie poblada de encinas que limitaban con el Cortijo de Árboles, la compra en este caso se le hizo a la propia Corona, ya que el rey Felipe II vendió a la orden estas tierras por 100.125 maravedíes, tal cantidad la había recibido el monasterio a cambio de una memoria encargada por el licenciado Luis Fernández de Córdoba ${ }^{56}$.

La compra de mayor cuantía de todas las que realizó el monasterio en el norte de Granada se produce a principios del siglo XVII, el año 1602 para ser exactos. Se trató del ya mencionado Cortijo Enciso, Cortijo Enciso, del cual ya hemos hecho alusión. Esta propiedad limitaba con en el camino de Granada a Alfacar y estaba cerca de otras fincas en Jun, Víznar y Pulianas que la orden venía comprando desde el año 1505. El gran cortijo adquirido a los hermanos Fernando, Sebastián y Juan Enciso Navarrete era una gran plantación de viña y olivar ${ }^{57}$. La extensión de la hacienda era de 3.600 marjales por la que se pagó

\footnotetext{
53 Ibidem, pp. 230-231.

54 Garzón Pareja, La orden de San Jerónimo en Granada, pp. 127-132.

55 La tabla ha sido elaborada según los datos aportados por el manuscrito de GARZÓN PAREJA, La orden de San Jerónimo en Granada y el trabajo de MARín LóPEz, “Origen y evolución...” y el libro 3.693 depositado en la Sección Clero del (AHN).

56 Rodríguez Molina, “Los jerónimos de Granada...”, p. 270.

57 (AHN), Sección Clero, lib. 3.693, fol. 216r.
} 
la gran suma de 1.800 .000 maravedíes $^{58}$. Pese a que los precios habían subido desde el siglo XVI, el altísimo importe desembolsado por el monasterio para la adquisición del Cortijo Enciso nos demuestra claramente el poderío económico del que hacía gala la casa granadina. Este conjunto patrimonial que contenía numerosas propiedades incluía varios censos, algunos de los cuales quedaron redimidos por los vendedores quedando para el monasterio un censo de 76 maravedíes que se pagaba a la parroquia de El Salvador ubicada en el Albaicín ${ }^{59}$. Esta finca, hoy conocida como El Ciso, se convirtió en el núcleo central de las propiedades que los jerónimos tenían en el norte de la ciudad, atravesadas por el camino de Alfacar que abarcaba toda la zona alomada en dirección Víznar.

El último cortijo del que haremos mención estaba ubicado en Sierra "Sulayr" "60 su nombre, homónimo al de la orden, y que aún existe, es el cortijo de San Jerónimo, localizado al comienzo de la dehesa del mismo nombre en el margen del río, a una legua de Monachil ${ }^{61}$. Desconocemos si el cortijo estaba ya construido cuando la orden recibió los prados de la sierra en 1495, ya que las referencias a esta propiedad son escasas. Aunque sí tenemos constancia de que se empleó como cabaña ganadera y para la siembra del cereal, el rendimiento del cortijo oscilaba entre ocho y diez fanegas de producción anual ${ }^{62}$.

\section{La evolución y desarrollo de las propiedades de la orden en Granada y su hin- terland (Ss. XV-XVII)}

La política de acumulación de propiedades que hemos visto en torno a una finca donada o adquirida por el monasterio, también se llevó a cabo en la urbe y en su hinterland. Aunque en este caso tuvo menor entidad que en las otras zonas ya analizadas. Lógicamente en un entorno urbano había menos oportunidades de monopolizar la propiedad del espacio, a lo que debemos sumar que, tras la conquista de la ciudad, muchos de los participantes en la Guerra de Granada obtuvieron mercedes y bienes en torno a la ciudad $^{63}$. Por lo tanto, el margen de maniobra para la orden fue más estrecho debido a la escasez de buenas propiedades, mientras que las almunias ubicadas en el entorno de la ciudad fueron parceladas y repartidas siendo la principal preocupación para los hermanos la obtención de la huerta de Dar Abenmordí para la edificación del monasterio ${ }^{64}$.

58 (AHN), Sección Clero, lib. 3.693, fol. 216r.

59 Marín López, “Origen y evolución...”, p. 233.

60 Nombre en árabe para Sierra Nevada, en la documentación suele aparecer como "sierra çolera".

${ }^{61}$ Las dehesas que disfrutó la orden tras la conquista del reino nazarí habían pertenecido a la familia real nazarí, allí pastaba el ganado de los propios emires, Espinar Moreno, "Ganados y pastos en Sierra Nevada. La dehesa del Junín", pp. 267-282.

62 De Rojas Clemente Rubio, Viaje a Andalucia "Historia Natural del Reino de Granada (1804-1809)", p. 933 y p. 1.099

63 Para ver las donaciones y mercedes reales realizadas en Granada tras su conquista ver LADERo QUESADA, "Mercedes reales en Granada anteriores al año 1500", pp. 355-424.

64 IBN AL JATIB: Historia de los reyes de la Alhambra, pp. 104-105. El célebre polígrafo granadino menciona más de 30 almunias en Granada y su hinterland. 
Desde finales del siglo XV la orden acumula propiedades cercanas al lugar donde estaban asentados. La huerta de Martín Guerra fue adquirida en la primera mitad del siglo XVI, en la documentación manejada no aparece el recibo de la transacción, sólo sabemos que lindaba con la huerta del monasterio. Otra propiedad en posesión de los jerónimos es la Huerta de los Almeces, que había sido comprada por Diego de Sevilla en 1493 y que aparece en posesión del monasterio a principios del siglo $\mathrm{XVI}^{65}$. En este caso tampoco tenemos información de cómo la citada huerta acabó en posesión del monasterio granadino. Lo único que sabemos a partir de las actas capitulares de las casas es que "la Huerta de los Almezes, que está junto a nuestro Hospital que llaman de Juan de Dios" y la pequeña "Güerta de San Juan", anexa al monasterio, en la cual se plantaron viñas, en el año 1567 los jerónimos se negaron a venderlas como solares pese a que la ciudad se extendía a su alrededor ${ }^{66}$.

La huerta de la Almoraba donde la orden vivió entre 1492 y 1521, en la antigua mezquita de Maharoch, llamada en castellano Ermita del Quemado, estuvo en posesión del monasterio hasta 1544, año en que fue donada por los jerónimos para la construcción del Hospital de San Juan de Dios ${ }^{67}$.

Los hornos, almazaras y molinos de cereal que fueron entregados en las distintas dotaciones reales permanecen durante el periodo analizado en posesión del monasterio. Es más, su número se vio aumentado por la donación realizada mediante diferentes fundaciones pías de distintos molinos de trigo y aceite en Cubillas, Moclín, Cújar y Genil ${ }^{68}$. La única excepción fue el horno de Guimien que, como ya hemos visto, fue vendido y que era parte de la primera dotación del año 1495. Estaba ubicado en el barrio de "Rabad Bucaralfacin" y perteneció a los jerónimos hasta el año 1608 en el que se traspasó al Hospital de San Juan de Dios por 1.200 ducados. El dinero de la transacción fue invertido en la compra de una finca de viñedos y olivar situada en el término de Cújar. El antiguo propietario de esta propiedad era Diego de Alcaraz ${ }^{69}$. Apreciamos que más de un siglo después de la fundación, los monjes seguían mercadeando con algunos de los bienes que les habían sido donados a la orden en la primera dotación regia para comprar propiedades que resultaban más afines a sus intereses según el momento y la necesidad.

La venta del horno de pan al Hospital de San Juan de Dios puede deberse a la necesidad del sanatorio de disponer de este producto para la alimentación de los enfermos. Necesidad en este caso aprovechada por el monasterio para seguir consolidando su modelo latifundista, como puede verse en las adquisiciones de tierra en las proximidades de Cújar que se iniciaron en 1505, manteniéndose hasta el primer cuarto del siglo XVII. La última compra que los jerónimos realizan en la zona se produce en el año $1625^{70}$.

\footnotetext{
65 (ARCGR), sala 202, leg. 5.276, pieza 6, fol. 1r.

66 Rodríguez Molina, "Los jerónimos de Granada...”, p. 272.

67 Espinar Moreno, "De la Mezquita de Maharoch al Monasterio de San Jerónimo", pp. 74-75 y SÁnCHEZ Martínez, "Granada: Origen del Hospital de San Juan de Dios", p. 338.

68 Rodríguez Molina, “Los jerónimos de Granada...”, p. 269.

69 Marín López, "Origen y evolución...," p. 231.

70 Marín López, “Origen y evolución...”, p. 231.
} 
Otras propiedades que fue adquiriendo el monasterio mediante compra o por la fundación de capellanías fueron numerosas casas distribuidas por toda la ciudad. La cuantificación de las viviendas resulta una labor compleja debido al volumen y a la dispersión documental, una aproximación puede realizarse a tenor de la documentación contenida en el libro de censos del monasterio ${ }^{71}$. Dicho tomo arroja una cifra de 178 casas u otras propiedades urbanas y periurbanas. Entre las propiedades periurbanas encontramos numerosos cármenes, no solo en la ciudad de Granada y su hinterland, sino también en el Pago de Cújar, en el término de Víznar y los llamados cármenes del Genil "Xenil"?2. En referencia a las casas, su ubicación, es variada ya que encontramos la mayoría en torno a la calle de San Juan de Dios y el Zacatín, desde Bibrambla a Plaza Nueva, en la calle de Elvira, la Alhóndiga, Pescadería, "junto al Boquerón de Darro, en la calle que baxa de la Carneçería de la calle Elvira para Sancta Paula", "en la collaçión de Santiyuste", y en el Campo del Príncipe. Conocemos la existencia de varias propiedades en otros núcleos urbanos de las que los jerónimos tuvieron posesión: podemos mencionar una casa en Alcalá la Real y un mesón en la Malahá (Localidad ubicada en la región del Quempe ${ }^{73}$.

El estudio de las 178 propiedades que aparecen en el libro de censos nos aporta una valiosa información sobre el origen de las propiedades, 26 de ellas corresponden a la dotación de los Reyes Católicos y en su mayoría son tiendas. El resto proviene de la fundación de capellanías y memorias. El periodo que abarca el tomo son los siglos XVI y XVII, pese a que la documentación en ocasiones aparece incompleta y no nos indica el origen de la propiedad, la denominación del bien o el importe a percibir, no obstante, sí que conocemos el tipo de censo al que estaba sujeta. En este punto debemos realizar una distinción, en primer lugar, encontramos alquileres que suelen corresponder a propiedades adquiridas directamente por el monasterio. En la documentación analizada este tipo de contrato corresponde en su mayor parte a las tiendas que fueron donadas al monasterio ${ }^{74}$. Los censos propiamente dichos tienen tres tipologías: abierto, perpetuo y redimible ${ }^{75}$. La documentación analizada en especial los libros de censos de la orden puede ocasionar errores, ya que en ocasiones aparece la palabra "alquiler" para denominar un censo, para evitar equivocaciones debemos remitirnos, siempre que aparezca especificado, al origen de la propiedad. Pese a que el fin es similar y el monasterio percibe una cantidad de renta previamente fijada por la propiedad, la entidad jurídica de los bienes provenientes de una capellanía o memoria es distinta, ya que pasan a convertirse en bienes espirituales y son inalienables. A excepción de los censos redimibles, que suelen tener

\footnotetext{
71 (AHN), Sección Clero, lib.3392. s/f.

72 Las propiedades denominadas cármenes del árabe "karm”, eran pequeñas almunias o fincas aristocráticas, situadas en el entorno periurbano de la ciudad con ricos huertos y viñas posiblemente destinadas al comercio. Sobre los cármenes de Aynadamar, los más famosos de la ciudad de Granada, habla BARRIOS Aguilera, "Paisajes agrarios moriscos en Granada", pp. 217-238 y Argente del Castillo Ocaña, "La vivienda granadina”, pp. 137-157.

73 Rodríguez Molina, "Los jerónimos de Granada...," p. 269.

74 (AHN), Sección Clero, lib. 3.392. s/f.

75 Para conocer las características propias de cada una de las tipologías de censos existentes, podemos recurrir al trabajo de Moreno TrujlLlo, "El Documento de Censo en la Castilla del Siglo XVI".
} 
origen en un préstamo realizado por la orden a un particular, tras el pago de la deuda, el particular puede recuperar la propiedad pagando al monasterio la cantidad debida. En ocasiones estas propiedades sujetas a censos redimibles acaban en posesión de la orden por impago, al igual que los censos abiertos y perpetuos que permanecieron en posesión de las distintas órdenes regulares hasta la desamortización.

Estos inmuebles contaban además con el principal hándicap añadido de la pérdida de valor progresiva, ya que, en numerosas ocasiones, principalmente las casas y otras propiedades urbanas acaban en ruinas, debido a que las órdenes no solían invertir dinero en rehabilitación o reformas y sólo buscaban percibir la renta fijada en la fundación de la capellanía o memoria. Hay que tener en cuenta que la mayoría de los censos que aparecen para el caso de los jerónimos son perpetuos, de las 178 propiedades estudiadas, 21 tienen su origen en la dotación de los Reyes Católicos, 38 son de régimen abierto, 20 son redimibles, no consta su origen en 9 y los 90 restantes son perpetuos. El total aproximado que percibe la orden por las rentas de estas propiedades anualmente es de 4.024.323 maravedíes ${ }^{76}$. Aunque debemos especificar que es una cantidad aproximada, ya que para algunos bienes no contamos con la renta apercibida y el periodo temporal que aparece en el libro de censos abarca desde el año 1506 hasta el 1697. No obstante, nos da una idea de la magnitud e importancia de los ingresos provenientes de memorias, aniversarios y capellanías que engrosaban considerablemente las arcas del Monasterio de Santa María de la Concepción de la orden de San Jerónimo de Granada.

Volviendo a la lista de bienes que aparece en el libro de censos, encontramos por ejemplo que, pese a resultar una minoría, los inmuebles que pasan a ser propiedad de la casa granadina tras una fundación pía, sirven para la consolidación del modelo latifundista que desarrollaron los jerónimos. Un ejemplo de ello lo tenemos en dos haciendas en el pago del Nublo, en 1518 la orden recibe 18 marjales y un haza en el año $1657^{77}$. Otra haza fue donada en Dar Abenmordí, al lado del monasterio en el año 1646. Como hemos visto, estas propiedades estaban todas ubicadas cerca de la casa capitular. Aunque el fenómeno también aparece en las proximidades de otras zonas monopolizadas por la orden, al norte de la ciudad encontramos la Huerta de San Lázaro que pasó a ser posesión de los monjes en 1636. En los términos de Cújar y Víznar al norte y en el camino de la Zubia, cerca del pago del Nublo, apreciamos cómo en 1608 y 1639 se donan varias hazas. Al sur en Santa Fe reciben 200 marjales en 1564. Resulta interesante ver como las donaciones aparecen

76 MARÍN LÓPEZ, “Origen y evolución...”, p. 238.

77 Si atendemos al Tomo IV (1734) del diccionario de autoridades de la Real Academia Española (RAE) la definición para el término haza es: s. f. Propriamente se llama assí el campo donde se ha segado trigo o otra semilla, y que está ocupado de los haces y gavillas que han hecho los segadores: y también se llama assí una cierta porción de tierra, aunque no esté sembrada. Latín. Seges. Ager. HORTENS. Mar. f. 47. Hermosa es una haza de pan, assí ahora en berza, con aquel verdor desigual, en que casi tropiezan unas con oras las cañas. HERN. Eneid. lib. 12.

Cuyo mui pobre Padre tuvo a renta,

Ciertas estrechas hazas que sembraba.

Como podemos apreciar tras la descripción, la superficie de cada haza era variable y no tenían unas medidas establecidas, de esta forma no podemos conocer con exactitud la superficie de tierra que recibía el monasterio con cada una de las donaciones. 
incluso en zonas bastante alejadas de la capital, en 1649 reciben un haza en Berja, lugar donde la orden alquilaba dehesas de verano para el pastoreo de su cabaña ganadera, o un haza de caña dulce en Motril en el año 1606, dotando de esta manera al monasterio de un plantío que no se cultivaba en ninguna de las otras propiedades de las que disponía. La caña de azúcar sólo crecía en las cercanías del Mediterráneo, debido a que el clima en las zonas costeras es más suave que en el interior ${ }^{78}$.

\begin{tabular}{|c|c|c|}
\hline Año & Concepto & Cantidad (en maravedíes) \\
\hline 1494 & 150 marjales en Santa Fe & $\mathrm{n} / \mathrm{c}$ \\
\hline \multirow[t]{6}{*}{1495} & Cortijo de Ficullar & 15.000 \\
\hline & Prados de la Sierra & 15.000 \\
\hline & 47 tiendas & 60.000 \\
\hline & 600 fanegas de trigo y 400 de cebada & $\mathrm{n} / \mathrm{c}$ \\
\hline & 300 carneros & $\mathrm{n} / \mathrm{c}$ \\
\hline & 200 fanegas de sal & $\mathrm{n} / \mathrm{c}$ \\
\hline 1504 & Molino de aceite & $\mathrm{n} / \mathrm{c}$ \\
\hline \multirow[t]{3}{*}{1505} & Alcabalas en alhóndiga Zaida & 50.000 \\
\hline & Rentas: lana, especería, cueros, jabón y hagüela & 194.750 \\
\hline & $\begin{array}{l}1095 \text { fanegas de trigo repartidas entre las } \\
\text { alquerías de la Vega }\end{array}$ & $\mathrm{n} / \mathrm{c}$ \\
\hline 1525 & Capellanía del Gran Capitán & 50.000 \\
\hline 1589 & Capellanía de María Sarmiento & $80.000^{79}$ \\
\hline 1603 & Capellanía de Juan de Aragón & 35.000 \\
\hline \multirow[t]{8}{*}{ Finales s. XVII } & Capellanías, misas y memorias (Total) & 2.682 .001 \\
\hline & Casas (170) & 964.240 \\
\hline & Molino de papel & 51.000 \\
\hline & Juros (sin contar alcabalas de Castro del Rey) & 1.199 .284 \\
\hline & Huertas, riego y secano & 1.523 .954 \\
\hline & Viñas y olivar & 363.732 \\
\hline & Ganado & 941.970 \\
\hline & Total (aproximado) & 8.225 .931 \\
\hline
\end{tabular}

Tabla general sobre rentas de los jerónimos (Ss. XV-XVII) ${ }^{80}$.

\footnotetext{
78 Marín López, “Origen y evolución...”, pp. 235-238.

79 Esta capellanía se hizo sobre unas alcabalas de Castro del Rey; el dinero corresponde a un juro firmado por Felipe II a Pedro Fernández de Córdoba y Fernández de Córdoba IV marqués de Priego e hijo del Gran Capitán. El juro quedó en la familia y años después María Sarmiento, su nuera, funda en el monasterio una capellanía perpetua con una dotación de 80.000 maravedíes. Con motivo de ello, se hace una reformulación del juro a favor del monasterio. Toda la información se encuentra en el (AHPG) lib. 7743.

80 Los datos han sido extraídos del manuscrito de Garzón Pareja, La orden de San Jerónimo en Granada, del ensayo de Marín López, "Origen y evolución...", del trabajo de SÁnchez Carrasco, "Las dos dotaciones fundacionales...", de la obra de Espinar Moreno, "De la Mezquita...", del lib. 3.693 depositado en la Sección Clero del AHN y de los libros 1.252 y 7.743 depositados en el AHPG.
} 
Patrimonio del Monasterio de Santa María de la Concepción de la Orden de San Jerónimo de Granada (s.XV-XVI)

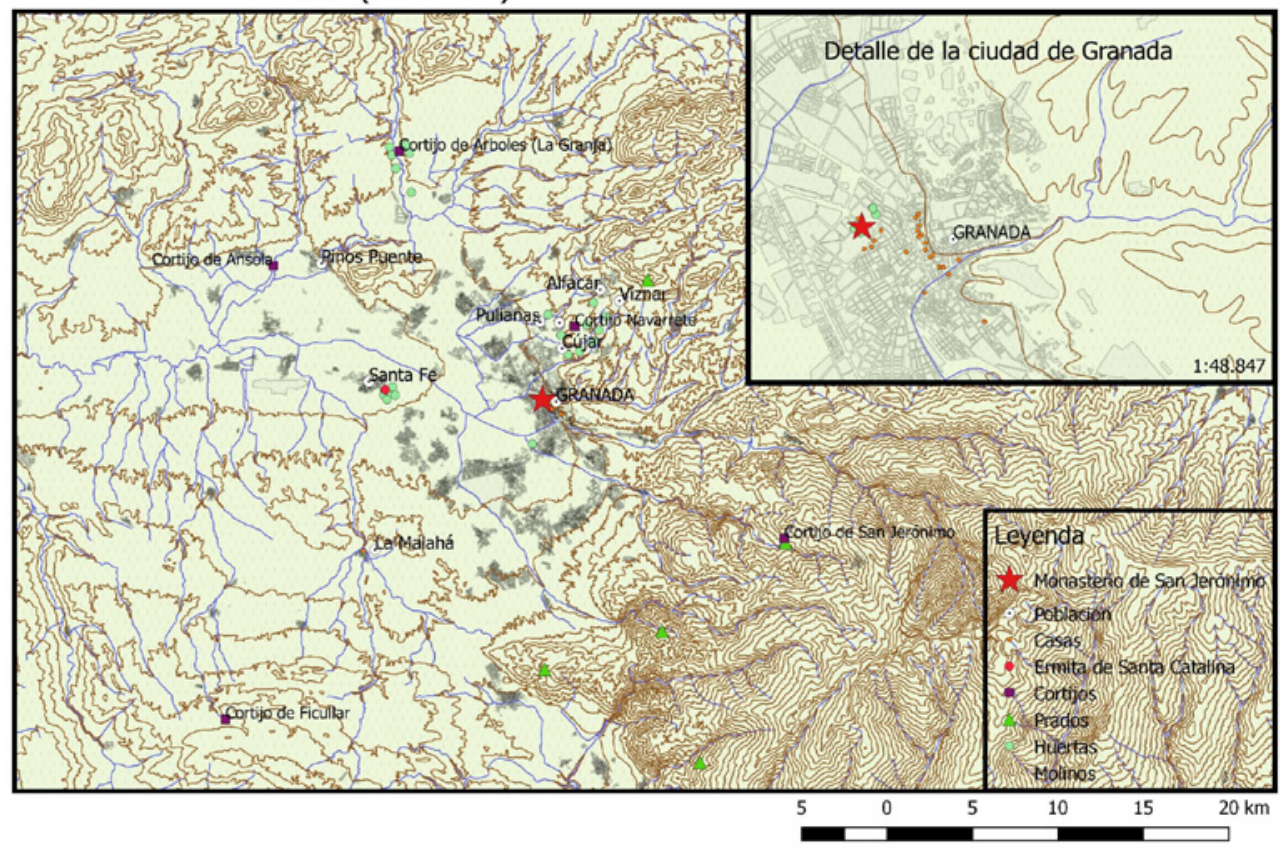

Mapa del patrimonio del Monasterio de San Jerónimo ${ }^{81}$.

\section{Conclusiones}

A nivel económico e institucional el despegue definitivo de la orden se produce en el año 1505 . Es cierto que ya habían mostrado cierta capacidad adquisitiva gastando miles de maravedíes en materiales edilicios, pero no es hasta la segunda y generosa donación que realizaron los Reyes Católicos cuando podemos afirmar que el monasterio granadino tuvo autonomía plena. En cómputos globales el montante de las dos donaciones superaba el medio millón de maravedíes, lo que era una auténtica fortuna y constituyó la dotación más grande que los monarcas hicieron a alguna de las órdenes monásticas que se afincaron en el recién conquistado reino nazarí. Ello indica que los reyes tenían un especial interés en contar con la presencia de la orden que era de patronato real. Durante el periodo analizado, los jerónimos funcionaron como una empresa manteniendo dos estrategias claramente definidas: minimizar los gastos en la medida de lo posible y concentrar sus posesiones en torno a varios focos concretos conformando verdaderos

\footnotetext{
81 Fuente: Elaboración propia, el programa empleado ha sido el QGIS y los datos han sido extraídos de: el manuscrito de Garzón PAREJA, La orden de San Jerónimo en Granada, del trabajo de Marín López, "Origen y evolución...", del trabajo de SÁnchez CARRASCO, "Las dos dotaciones fundacionales..." y del libro 3.693 depositado en la Sección Clero del AHN.
} 
latifundios en el ámbito rural y controlando el espacio urbano anexo a la casa de la orden siguiendo la misma estrategia de acumulación de propiedades.

Pese a que los jerónimos tuvieron posesiones a lo largo y ancho del reino de Granada e incluso fuera de él, podemos definir tres focos donde la orden concentró su interés tras obtener solvencia suficiente para realizar transacciones económicas. El primero de ellos fue en Santa Fe, en torno a la ermita de Santa Catalina. Desde 1505 hasta finales del siglo XVI adquieren y permutan toda la tierra de cultivo que pueden en este entorno, de elevado valor simbólico ya que fue el primer lugar que habitaron tras llegar a Granada. El segundo foco y el más importante estuvo constituido por las posesiones del monasterio en el norte de la ciudad. Allí, desde 1505 hasta mediados del siglo XVII, compran y permutan una gran cantidad de tierra gastando varios millones de maravedíes. El tercer foco y de menor tamaño estuvo configurado en el entorno del monasterio, la huerta de Dar Abenmordí, otrora ubicada extramuros, que era un lugar perfecto para la vida monástica. En este lugar, en primera instancia, acumulan las fértiles huertas de regadío cercanas al monasterio. En cambio, cuando el desarrollo urbano afecta al monasterio, durante la segunda mitad del siglo XVI, cambian de estrategia y empiezan a adquirir viviendas con el fin de crear un área de influencia en el recién construido barrio del que se constituyen como eje articulador.

Analizando la entidad de los antiguos propietarios de los bienes que poseyó la orden, podemos hacer una distinción en tres grupos según el periodo en el cual el monasterio tomó posesión de ellos. En primer lugar, los más antiguos, que pertenecían a los Reyes Católicos y que habían sido de la familia real nazarí o miembros distinguidos de su corte, están constituidos por huertas fértiles o tienen ricos recursos naturales y llegaron a la orden gracias a la donación real en la última década del siglo XV. Un segundo grupo está constituido por las propiedades que el monasterio compra en las áreas que eran de su interés entre el año 1505 y 1568 . Entre los antiguos propietarios de estas tierras encontramos a numerosos moriscos entre los que podemos destacar la presencia de personajes de distinto estatus socioeconómico, desde campesinos hasta comerciantes. Finalmente, las posesiones que engrosan el patrimonio de la orden tras la Guerra de las Alpujarras hasta el siglo XVIII provienen de cristianos viejos y son adquiridas por ella o donadas por fieles con amplia solvencia económica para la fundación de capellanías. Las redes clientelares que estableció el monasterio lo ayudaron a incrementar su patrimonio atendiendo a las oportunidades que se le presentaban en el mercado. Un ejemplo de ello lo tenemos con el Cortijo de Árboles o el Cortijo de Ansola, ambos adquiridos a un precio mucho más bajo del que tenían en realidad debido a las distintas vicisitudes que sufrieron sus antiguos propietarios. Existió un profundo nivel de planificación por parte del monasterio en torno a las distintas facetas económicas que explotaba, un ejemplo de ello lo tenemos en la introducción en sus tierras de cultivos con los que no contaban como olivos o viñas. El aumento de las cabezas de ganado de la cabaña ganadera de la orden y la necesidad de mejoras e infraestructuras en las tierras del monasterio, priorizó la movilidad de recursos y la adquisición de otras propiedades que fueran afines a sus intereses económicos. La compra de extensiones de secano en las que se construyeron 
cabañas para el ganado indica que fue la solución que se tomó para evitar el alquiler de pastos de invierno cuando las inclemencias climáticas impedían el pastoreo en las dehesas que la orden tenía en la sierra.

\section{Bibliografía citada}

Atienza LóPEz, Ángela, "Fundaciones frustradas y efímeras en la España moderna. Memoria de los conventos franciscanos que no pudieron ser", Cuadernos de Historia moderna, 39 (2014), pp. 189-209.

Atienza LóPez, Ángela, "El mundo de las monjas y de los claustros femeninos en la edad moderna. Perspectivas recientes y algunos retos", en De la tierra al cielo: Líneas de investigación reciente en historia moderna, coord. Eliseo serrano Martin, vol. 1, Fundación Española de Historia Moderna, Institución Fernando el Católico, Zaragoza, 2012, pp. 89-108. AtIENZA LóPEZ, Ángela, "Los que fracasaron: fundaciones y fundadores frustrados. La otra cara de la expansión conventual en la España Moderna", en Iglesia, poder y fortuna: Clero y movilidad social en la España Moderna, coord. Enrique Soria Mesa y Antonio José Díaz Rodríguez, Comares, Granada, 2012, pp. 89-114.

Argente del Castillo Ocaña, Carmen, "La vivienda granadina. Una aproximación a su tipología (1492-1516)", Cuadernos de estudios medievales y ciencias y técnicas historiográficas, XVIII-XIX, (1993-94), pp. 137-157.

Barrios Aguilera, Manuel, "Paisajes agrarios moriscos en Granada (a través de los Libros de Apeo)", Revista del Centro de Estudios Históricos de Granada y su Reino, 3, segunda época, (1989), pp. 217-238.

Espinar Moreno, Manuel, "Ganados y pastos en Sierra Nevada. La dehesa del Junín (Siglos XIV-XV)”, VII Estudios de Frontera. Homenaje a $M^{a}$ Jesús Viguera Molins, Diputación Provincial de Jaén, Jaén, 2008, pp. 267-282.

Espinar Moreno, Manuel, "De la Mezquita de Maharoch al Monasterio de San Jerónimo. Noticias para el urbanismo y la arqueología de Granada (1358-1505)”, Cuadernos de estudios medievales y ciencias y técnicas historiográficas, 18-19 (1993-1994), pp. 73-97. GARZÓn PAREJa, Manuel, La orden de San Jerónimo en Granada, manuscrito, Granada, 1975.

García Valverde, María Luisa, "El archivo del convento de agustinas recoletas del santísimo Corpus Christi de Granada a través de las visitas pastorales de los siglos XVII y XVIII", Memoria eclessiae, 15 (1999), pp. 471-486.

García Valverde, María Luisa, "El monasterio de Santa Isabel la Real de Granada: Su fundación y su archivo", Archivo Ibero-Americano, 231 (1998), pp. 491-530.

IBN AL JATIB, Historia de los reyes de la Alhambra (Al-Lamha al-Badriyya): Resplandor de la luna llena acerca de la dinastía nazarí, Universidad de Granada, Granada, 2011. 
LADERo Quesada, Miguel Ángel, "Mercedes reales en Granada anteriores al año 1500". Hispania, 111 (1969), pp. 355-424.

Luna Díaz, Juan Andrés, "Apuntes para el estudio de la agricultura de Granada y su tierra en los primeros años de la conquista", Chronica Nova, 10 (1979), pp. 231-248.

López Martínez, Antonio Luis, "El patrimonio rustico de los jesuitas en España: Una aproximación”, Hispania: Revista española de historia, 203 (1999), pp. 925-954.

López Martínez, Antonio Luis, "La empresa agraria monástica andaluza: gestión de las explotaciones agrarias de la Orden Cartuja (siglos XV-XIX)", Hispania: Revista española de historia, 196 (1997), pp. 709-729.

López Martínez, Antonio Luis, "El patrimonio económico de los jesuitas en el reino de Sevilla y su liquidación en tiempos de Carlos III", Archivo hispalense: Revista histórica, literaria y artística, 217 (1988), pp. 35-60.

Marín López, Rafael, "Origen y evolución del patrimonio del Monasterio de San Jerónimo de Granada (Siglos XVI-XVII)”, Chronica Nova, 26 (1999), p. 215-242.

Morell y Terry, Luis, Equivalencias métricas de la provincia de Granada, Gaceta del Sur, Granada, 1909.

Moreno Trujillo, María Amparo, "El Documento de Censo en la Castilla del Siglo XVI", Revista de Derecho Notarial, CXLV-CXLVI (1989), pp. 313-352.

Peinado Santaella, Rafael Gerardo, "Una aportación documental sobre el poblamiento, el paisaje agrario y la propiedad de la tierra de dos alquerías de la vega de Granada: Chauchina y el Jau a finales del período nazarí", Revista del Centro de Estudios Históricos de Granada y su Reino, 10-11 (1996-1997), pp. 19-92.

Peinado Santaella, Rafael Gerardo, La fundación de Santa Fe (1491-1520), Universidad de Granada, Granada, 1995.

Rodríguez Molina, José, "Los jerónimos de Granada. Procedencia de sus riquezas", Estudios sobre Patrimonio, Cultura y Ciencias Medievales, 13-14 (2012), pp. 263-287.

Rojas Clemente Rubio de, Simón, Viaje a Andalucía "Historia Natural del Reino de Granada" (1804-1809), Griselda Bonet Girabet, Almería-Barcelona, 2002.

Romero Martínez, Adelina, "Documentos para la historia del arte granadino. El Monasterio de San Jerónimo", en Estudios sobre iglesia y sociedad en Andalucía en la edad moderna, Miguel Luis López-Guadalupe y Antonio Luis Cortes Peña (coords.), Universidad de Granada, Granada, 1999, pp. 505-520.

SÁnchez CaRrasco, Juan José, "Las dos dotaciones fundacionales del Monasterio de Santa María de la Concepción de Granada", Espacio-tiempo y forma, Serie III, Historia Medieval, 30 (2017), pp. 505-537.

SÁnchez Herrero, José, "Fundación y Desarrollo de la Orden de los Jerónimos, 13601561", Codex aquilarensis: Cuadernos de investigación del Monasterio de Santa María la Real, 10 (1994), pp. 63-95. 
SÁnchez Martínez, José, "Granada: Origen del Hospital de San Juan de Dios en las fuentes jerónimas (1520 - 1544)", Archivo Hospitalario, 4 (2006), pp. 375-398.

TriLlo SAN José, Carmen, "El paisaje vegetal en la Granada Islámica y sus transformaciones tras la conquista castellana", Historia Agraria, 17 (1999), pp. 131-152.

Trillo SAn JosÉ, Carmen, "Fiscalidad mudéjar en el reino de Granada: las rentas del Quempe", Anuario de estudios medievales, 22 (1992), pp. 855-857.

Valladar y Serrano, Francisco de Paula, "El Patronato del Gran Capitán en la Iglesia de San Jerónimo", La Alhambra, año 4, tomo IV, nueva época, (1901), pp. 355-356.

Valladar y Serrano, Francisco de Paula, "La Iglesia de San Jerónimo", La Alhambra, año 9, tomo IX, nueva época, (1906), pp. 363-367 y 433-437.

Vera Delgado, Ana María, "La Revuelta Mudéjar de 1500-1501: El destino de los vencidos", en Andalucía medieval. Actas del I Congreso de Historia de Andalucía, diciembre de 1976, vol. 2, Publicaciones del Monte de Piedad y Caja de Ahorros de Córdoba, Córdoba, 1978, pp. 387-394. 
